\title{
SUPPLEMENTATION OF MICRONUTRIENT IN COMMUNITY MICRONUTRIENT DEFICIENCY PREVENTION PROGRAMMES
}

\author{
M.D. Dairo and O.K. Ige
}

Department of Epidemiology \& Medical Statistics, Faculty of Public Health, College of Medicine, University of Ibadan, Nigeria.

Correspondence:

Dr. M.D. Dairo

Department of Epidemiology \& Medical Statistics, Faculty of Public Health,

College of Medicine,

University of Ibadan,

Nigeria.

E-mail:dairomagbagbeola@yahoo.com

Tel: 08035664708

\begin{abstract}
Micronutrient deficiency affects about 2 billion people all over the world. The major micronutrient deficiencies which are of public health importance include vitamin $A$, iron and iodine deficiencies. The deficiencies of these micronutrients cause a variety of morbidities and increased mortality which are most severe in children, adolescent girls and pregnant women. Despite the magnitude of these deficiencies, research has shown that they are correctable using simple strategies. This review examines the strategies employed to reduce micronutrient deficiencies worldwide by reviewing bibliographic databases, monograms and journals up to the year 2007. These strategies include food based and micronutrient supplementation. Although micronutrient supplementation is widely embraced as a strategy to combat micronutrient deficiencies, they are most suitable when used as a measure to combat severe deficiencies. Food based strategies such as food fortification and dietary diversification are more effective than micronutrient supplementation which should only be used as a short term measure. Supplementation programmes should be evidence based, multi-nutrient in approach and backed up with other complementary public health interventions.
\end{abstract}

Keywords: Supplementation, iron, vitamin A, iodine, combined strategy

\section{INTRODUCTION}

Micronutrient deficiencies are becoming more widespread especially in those countries in the developing part of the globe. Micronutrient malnutrition affects approximately 2 billion people worldwide ${ }^{1}$. Nutritional deficiencies have become more prevalent following economic stress and food insecurities faced by populations in these countries. Most at risk groups include children less than 5 years of age, adolescents, women of childbearing age, particularly the pregnant and lactating, refugees and victims of famine.

Vitamin A, iodine and iron deficiencies are of major public health importance in most parts of Africa and have been the cause of widespread mortality and morbidity in children, adolescents and women on the continent of Africa. These deficiencies have caused decreased productivity and loss of revenue to individuals and governments in Africa. Perhaps the continued poverty of the peoples of the continent can be traced to these deficiencies. Community prevention of these deficiencies is effective, feasible and simple. Efforts need to be intensified to combat these deficiencies. Nutritional scientists and public health experts need to devise and define strategies to combat these problems frontally and to salvage Africans from these preventable deficiencies.

\section{Rationale for Supplementation}

Despite the consensus that food based approach is the recommended strategy for the prevention of micronutrient deficiency, literature provides the basis for the adoption of supplementation programmes in certain circumstances, such as in cases of severe deficiencies. Evidence has shown that the rate of conversion of â-carotene in fruits and vegetables to retinol is less than desired (1:12 in fruits and $B$ carotene and 1:24 in other carotenoids and leaves). Thus young children cannot eat enough fruits and vegetables to meet their Vitamin A requirements. This limits the 
effectiveness of dietary diversification as a means of delivering Vitamin $\mathrm{A}^{2}$. West and colleagues (1999) showed in a double blind cluster randomized trial that weekly low dose Vitamin A supplementation $(7000 \mu \mathrm{g}$ of retinol equivalents) in malnourished pregnant women in Nepal reduced maternal mortality by $49 \%$. Thus weekly supplementation could have a major effect on mortality in pregnant women in developing nations. ${ }^{3}$ Sommer and colleagues, in the 1980's demonstrated in a randomized controlled trial in Aceh Province Indonesia that Vitamin A supplementation in preschoolers reduced mortality by $34 \% 0^{1}$. Indian studies revealed that six monthly dosing of children between the ages of 6-11 months with Vitamin A (100, 000 I.U) and children 12-59 months (200,000 I.U) has been found to lower the incidence of keratomalacia by about $80 \% .^{4,5}$ A community trial of Vitamin A administration alongside polio vaccine in house to house campaigns showed that the incidence and severity of measles infection reduced following administration ${ }^{5}$.

\section{METHODS}

\section{Vitamin A Supplementation}

The periodic distribution of high dose Vitamin A supplements is the most widely applied intervention with proven effectiveness for treatment, prevention and control of Vitamin A deficiency. ${ }^{6} \mathrm{~A}$ minimum of $65 \%$ coverage in an iterative of 4-6 months vertical programs sustained over a period of at least three years is required to achieve results in the population. The strategy involves the universal administration of high dose vitamin A to all children regardless of their vitamin A status. This is given as a dose of 100,000 international units for children 6-11 months and 200,000 international units for children 12-59 months. This regimen is given at 4-6 monthly intervals ${ }^{6}$.

Since Vitamin A deficient individuals are not sought out, at risk children in the population are often missed. Thus the approach is best used to control Vitamin A Deficiency in highly prevalent areas. Yet experiences have shown in India that mass administration of Vitamin A supplements alone will not control xeropthalmia in the population for operational rather than physiological or biological reasons ${ }^{5}$. Furthermore, the programmes are not cost effective ${ }^{5}$.

Thus targeted distribution and low dose supplements which can be linked to sustainable multiple delivery channels within the community are being advocated. The distribution of high dose Vitamin A to targeted high risk groups such as neonates, malnourished children, those ill with measles, pneumonia and diarrhea has been proposed. The rationale for this is based on the evidence from studies in India and Indonesia although the findings have not been replicated in other countries. In India for example, administration of 24,000 I.U to newborns within 48 hours of birth produces a $22 \%$ reduction in early infant mortality. A similar result was observed in Indonesia where 64\% reduction in infant mortality is observed with the administration of 50,000 I.U of Vitamin A. ${ }^{7,8}$

A novel method of administering Vitamin A supplementation has been its concomitant administration during the Expanded Programme on Immunization routine and national immunization days. Experiences from India, Bangladesh and Nigeria reveal that this practice has been found to provide high coverage in several communities. In the house to house Immunization campaign in Nigeria, supplementation with Vitamin A has provided added advantages: communities with poor coverage of routine immunization, concomitant administration of Vitamin A capsules and polio or measles vaccines to children less than 5 years of age has increased the uptake of the latter in communities with previously low coverage of routine immunization ${ }^{5}$

Low Dose Supplement Programmes: These are best implemented through community systems which include trained voluntary health workers, women groups, pharmacies or patent medicine stores. Low dose supplement programmes designed to reach vulnerable groups on a weekly or monthly basis have been explored by Rahmatullah and colleagues and coverage of $90 \%$ was achieved within one year using trained part time community health volunteers ${ }^{4,5}$.

\section{Iron supplementation}

The traditional approach to combating iron deficiency is supplementation particularly during pregnancy. However, coverage and compliance rates are usually very poor ${ }^{9}$. Daily supplementation for pregnant women and women of childbearing age with 200 $300 \mathrm{mg}$ iron tablets has been found effective provided that compliance is adequate. Intermittent dosages either once or twice weekly have been tried and found to be equally effective, improves compliance and will also reduce expenses. ${ }^{10}$ Sloan et al. (1992) recommends that as a public health approach, prolonged supplementation beginning before the woman becomes pregnant may be a better strategy to benefit the majority of the population ${ }^{11}$.

\section{Iodine supplementation}

The advent of universal salt iodization has practically removed the need for administration of iodine supplements. Historically, the study that demonstrated that goiter can be prevented with ingestion of iodine involved the administration of sodium iodide to school children ${ }^{1}$. Lugols iodine and tablets of iodide 
salts can also be used. Iodinated oils taken orally or intramuscularly and Lugols iodine have also been successfully used in prevention programmes in the past. Iodized oils administered by injection is effective for three years, however, logistics of mass administration militates against its widespread adoption. Orally taken iodized oil is only effective for one year so its efficacy has not been established in public health terms. Other strategies for delivering iodine supplements include the iodination of community water supply and irrigation water.

\section{Limitations}

Given the necessity of repeated sessions required to achieve the expected result Vitamin A supplementation in prevention programmes, its sustainability cannot be guaranteed. At risk groups may be missed in mass supplementation programmes. Many at risk groups reside in geographical inaccessible areas and targeted supplementation may not be feasible because it operates through the health care system which is also faced with inadequate accessibility especially by the same at risk groups. Furthermore iron supplementation carried out in pregnancy does not include non pregnant pubertal girls who are also a high risk group. Compliance of pregnant teenage girls with iron supplementation regimes has been shown to be lower than for other women of child bearing age ${ }^{8}$ leading to a higher prevalence of anaemia in the former group.

\section{RECOMMENDATIONS}

The challenge to convince and win the support of policy makers, government functionaries and donors cannot be overcome without data. Studies on the magnitude of these deficiencies in Africa are imperative. The data on the prevalence of micronutrient deficiencies in countries in Africa is not well documented and oftentimes data are not available. Yet such data are not only required for advocacy for support but also for planning, implementation and monitoring of supplementation programmes. Data where available should be disaggregated by administrative divisions to enable planning and implementation of community based intervention programmes since implementation of supplementation programmes can only be based on accurate data.

Strategies that utilise established accessible, sustainable and culturally acceptable systems must be developed to increase the coverage of supplementation programmes. This is necessary to increase accessibility especially by high risk groups who often cannot benefit from existing systems.

Programs for intervention need to incorporate multinutrient supplementation strategies to optimize the use of scarce resources. Past intervention efforts have focused on reducing the prevalence of single micronutrient deficiency. However, multinutrient deficiency is the rule rather than the exception in communities where micronutrient deficiencies are found.

Although much evidence has been adduced to support the adoption of supplementation strategies, it must not be forgotten that micronutrient deficiencies occur in the context of deprivation. Thus supplementation should be combined with other strategies such as food fortification and dietary diversification to achieve the desired result. Supplementation should be adopted with the objective of reducing the severity of the deficiency and moving the community or country to the level in which food based strategies such as food fortification and dietary diversification, will become effective. Supplementation is the method of choice when therapeutic intervention is necessary - that is to address severe micronutrient deficiency. Supplementation is also an appropriate tool for preventive programs as long as those receiving the supplements consume them. Complementary public health interventions may be necessary to eliminate deficiency, such as combining mass measles immunization and Vitamin A supplementation, combining Zinc supplementation with Vitamin A supplementation, enhancement of Vitamin A status through diarrhoea disease control, and coupling deworming with iron supplementation to control anaemia.

There is a need for continued research into the most efficient and effective method for the delivery of micronutrient supplementation. As shown by Dairo and Lawoyin, those who need the supplements most comply the least ${ }^{9}$. It is not only necessary to distribute the supplements, we must assure their consumption. Research efforts should be directed to determine what factors affect compliance so that the compliance rate among those at risk of micronutrient deficiency can be improved. Furthermore, while the benefits of this supplementation regimen have been documented in community trials, data is still inadequate to evaluate the real benefits upon the community in many of the programmes. Thus data for advocacy is lacking and political support and will may be difficult to generate among the leadership of the micronutrient deficient communities.

\section{CONCLUSION}

Efforts should be accelerated towards food based strategies, while periodic supplementation should be combined in the interim with these food fortification and dietary diversification intervention programs. Such 
supplementation programmes should be evidence based, multinutrient in approach and backed up with other complementary public health interventions.

\section{REFERENCES}

1. Christopher P. Howson, Eileen T. Kennedy and Abraham Horwitz (Eds) Prevention of micronutrient deficiencies: Tools for Policymakers and public Health workers. http:/ / www.nap.edu/ catalo/5962.html p1.

2. Clive E. West and Ans Ellander, Bioefficacy of carotenoids in the Sight and Life Newsletter, Feb 2000. Culled from "Implications of research findings for programs to reduce micronutrient deficiencies "MOST. The USAID Micronutrient Program December 2003

3. West K.P. et al: Double blind cluster randomized trial of low dose supplementation with Vitamin A or Beta carotene on mortality related to pregnancy in Nepal. BMJ, 1999 Feb 27: 318(7183):570-575

4. Rahmatullah L., Underwood, B.A. Thulasiraj R.D., Milton R.C., Ramaswany K., Rahmatullah R. and Babu G. 1990: Reduced mortality among children in southern India receiving small weekly dose of Vitamin A. N. Eng J. Med 323: 929-935

5. Rahmatullah L., Underwood, B.A. Thulasiraj R.D., Milton R.C., 1991 Diarrhoea, respiratory infections and growth are not affected by weekly low dose Vitamin A supplement: a masked controlled field trial in children in southern India. Am J. Clin Nutr. 54: 568-577

6. Humphrey J.H., et al. Impact of neonatal Vitamin A supplementation on infant morbidity and mortality. J Pediatr. 1996 Apr;128(4):489-496.

7. Underwood B.A., Prevention of Vitamin A deficiency in Prevention of Micronutrient deficiencies: Tools for policymakers and public Health Workers p136. National Academy of Sciences. http://www.nap.edu/catalo/5962.html $\mathrm{p} 1$

8. Rahmathullah L., et al. Impact of supplementing newborn infants with Vitamin A on early infant mortality: community based randomised trial in southern India. BMJ. 2003 Aug 2;327 (7409):254.

9. Dairo, M.D. and Lawoyin T.O. Demographic factors affecting compliance with iron supplementation in pregnancy. Nigerian Journal of Medicine.

10. Ian Darnton-Hill: Control and Prevention of micronurient malnutrition. Asia Pacific J. Clin Nutr (1998) 7 (1): 2-7

11. Sloan N.L.E., Jordan A. and Winikoff B.,1992. Does iron supplementation make a difference? Mother Care Project, Working Paper 15. Arlington Va. 